\title{
Fitness efficacy of vibratory exercise compared to walking in postmenopausal women
}

\author{
Armando M. Raimundo • Narcis Gusi • \\ Pablo Tomas-Carus
}

Accepted: 17 April 2009

(c) Springer-Verlag 2009

\begin{abstract}
In this study, we compared the efficacy of 8 months of low-frequency vibration and a walk-based program in health-related fitness. Twenty-seven postmenopausal women were randomly assigned into two groups: whole-body vibration (WBV) group $(n=18)$ performed three times/week a static exercise on a vibration platform $(6$ sets of 1-min with $1 \mathrm{~min}$ of rest, with a $12.6 \mathrm{~Hz}$ of frequency and an amplitude of $3 \mathrm{~mm}$ ); walk-based program (WP) group $(n=18)$ performed three times/week a 60 -min of walk activity at 70-75\% of maximal heart rate. A healthrelated battery of tests was applied. Maximal unilateral concentric and eccentric isokinetic torque of the knee extensors was recorded by an isokinetic dynamometer. Physical fitness was measured using the following tests: vertical jump test, chair rise test and maximal walking speed test over $4 \mathrm{~m}$. Maximal unilateral isokinetic strength was measured in the knee extensors in concentric actions at 60 and $300 \%$, and eccentric action at $60 \%$ s. After 8 months, the WP improved the time spent to walk $4 \mathrm{~m}(20 \%)$ and to perform the chair rise test $(12 \%)$ compared to the WBV group ( $P=0.006,0.002$, respectively). In contrast, the comparison of the changes in vertical jump showed the higher effectiveness of the vibratory exercise in $7 \%(P=0.025)$. None of exercise programs showed change on isokinetic measurements. These results indicate that both programs
\end{abstract}

A. M. Raimundo $(\square) \cdot$ P. Tomas-Carus

Health Science and Technology Research Centre,

Department of Sport and Health, University of Évora,

Rua Reguengos de Monsaraz 14, 7000-727 Évora, Portugal

e-mail: ammr@uevora.pt

N. Gusi

Faculty of Sport Sciences,

University of Extremadura, 10071 Cáceres, Spain differed in the main achievements and could be complementary to prevent lower limbs muscle strength decrease as we age [ISRCTN76235671].

Keywords Aging $\cdot$ Fitness $\cdot$ Whole-body vibration $(\mathrm{WBV}) \cdot$ Muscle strength

\section{Introduction}

A major problem associated with advanced adult age is the remarkable decline in functional capacity and the associated loss of independence (Frontera and Bigard 2002). Muscle strength of the lower extremities is a major neuromuscular determinant of these losses and mobility (Guralnik et al. 1995). The loss of muscle strength starts to affect relevantly the quality of life around 50 years of age, and may compromise activities of daily living, especially in postmenopausal women because of the pronounced hormonal decline (Kallinen and Markku 1995). Longitudinal studies show a loss of approximately $1-2 \%$ per year in isokinetic strength of the knee (Frontera and Bigard 2002). In addition, it has been suggested that the product of force and speed is critical for reacting and preventing stumbling becoming a fall (Runge et al. 2000). However, adequate physical activity is an effective strategy for preventing this aging process (Frontera and Bigard 2002). To prevent this decline in strength, researchers have been mainly studying the dose-response of the most common resistance training programs such as weight lifting (McCartney et al. 1995). Even though this kind of exercise is suitable for young people, it is difficult to apply to frail persons (fallers, osteoarthritis, physically untrained elder, etc.), who are not used to use them (Yamazaki et al. 2004; Cardinale and Wakeling 2005). For this reason, walking-based programs have 